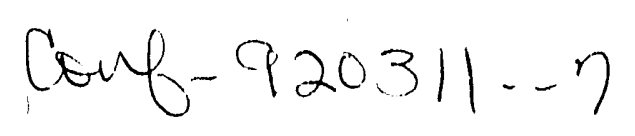

10 th Conference on Plasma Surface Interactions in Controlled Fusion Devices, March 30 - April 3, 1992, Monterey, CAL

Poster A:35

PPPL-CFP-2602

DE92 012672

M

\title{
PARTICLE BALANCE IN A TFTR SUPERSHOT
}

R.V. Budny, D. Coster, D. Stotler, M. G. Bell, A.C. Janos, and D.K. Owens

Plasma Physics Laboratory, Princeton University, P.O. Box 451, Princeton, NJ 08543, USA

Particle balance in a TFTR supershot is studied self-consistently. The TRANSP analysis code is used to model plasma parameters within the last closed flux surface, deriving time-dependent plasma profiles from measurements. The poloidal flux surfaces are derived using TRANSP and an equivalent-filament analysis code which distributes axisymmetric currents to match measurements of the poloidal field and flux and the total plasma current. The plasma in the edge and scrape off regions are modeled during a relatively steady state phase of the neutral beam injection using the $\mathrm{B} 2$ code which calculates plasma profiles in 2 dimensions. The recycled hydrogenic neutrals from the limiter are modeled with the DEGAS neutrals code. The recycling rates within the last closed flux surface are input into TRANSP. The edge and scrape off modeling results are compared with those from TRANSP in the main plasma and with measurements of the $D_{\alpha}$ emission and thermocouple measurements of temperature increases in the inner limiter. The recycling coefficients at the last closed flux surface and at the limiter are discussed. 


\section{Introduction}

It is important to characterize plasma in the edge and scrape off regions in tokamaks for various reasons. The heat and particle fluxes to the first walls are determinod partly by these regions and partly by charge exchange from the main plasma. Flasma properties in the main plasma region are influenced by these regions, and can have important consequences for the main plasma, such as determining the coupling of auxiliary heating to the main plasma and transport from the plasma back into the scrape off. Also in DT operation, the flux of $T$ to the walls will be partly determined by the scrape off. Since the tritium inventory for the TFTR DT experiments will be very limited, it will be important to assess and if possible, to limit this flux.

The scrape off regions in tokamaks are poorly known since they are generally neither poloidally nor toroidally symmetric. This means that comprehensive measurements would be needed to accurately characterize them, and that accurate modeling may have to be performed in 3 dimensions.

We study a TFTR supershot (\#55851) with $25 \mathrm{NiW}$ of NBI which performed well (yielded a high neuiron rate and exhibited no MHD), and which had extensive diagnostic coverage. The plasma current was $1.6 \mathrm{MA}$, the toroidal field $5.1 \mathrm{~T}$, the major radius $\mathrm{R}=$ $2.45 \mathrm{~m}$, and the minor radius, $\mathrm{a}=0.8 \mathrm{~m}$. The wall was well conditioned for this discharge, and a $\mathrm{Li}$ pellet was injected early (at $2.0 \mathrm{sec}$ ) to improve the plasma performance. 1 TRANSP modeling of this discharge has been described. ${ }^{2}$ The total numbers of particles within the last closed flux surface (LCFS) are shown in Fig. 1. We model the relatively steady state phase of the supershot near the end of the neutral beam injection (NBI). This paper discusses the deuterium and electron particle balance, the 
particle fluxes to the limiter, and the recycling of the deuterium from the limiter. The goals are to increase understanding of the edge, scrape off, and recycling.

The plasma regions of interest are shown schematically in Fig. 2. The plasma within the LCFS is divided into a main plasma region where the NBI is the dominant source of electrons and $D^{+}$, and an edge region where recycled $D^{0}$ from outside the LCFS is the dominant source. The region outside the LCFS is the scrape off region with plasma flowing to the limiter. The source rate in the main plasma is

$$
S_{m}=\frac{P_{N B}}{E_{N B}}=0.15 \times 10^{22} / \mathrm{sec}
$$

The $D^{+}$flux from the main plasma into the edge is

$$
\Gamma_{\mathrm{m}}=\mathrm{S}_{\mathrm{m}}
$$

and the flux from the edge into the scrape off is

$$
\Gamma_{\text {LCFS }}=S_{m}+S_{e d}
$$

The $D^{+}$flux onto the limiter is

$$
\Gamma W=S_{m}+S_{e d}+S_{s o}
$$

A fraction of this flux, $R_{W}$ is assumed to return as $D^{0}$ and the fraction $\left(1-R_{W}\right)$ is absorbed by the wall. If the particle balance were in steady state, the amount absorbed by the wall 
would be $S_{m}$. Figure 1 shows that the plasma is not in equilibrium to this accuracy since $S_{\text {ed }}$ varies.

The recycling coefficient $R_{W}$ is a measure of the limiter and wall conditioning. It is estimated by studying electron density decay after termination of gas puffing in ohmic discharges. The ratio of this decay time $\tau^{*}$ and the global particle confinement time $\tau_{D}$ gives $R_{W}$. Unfortunately $\tau_{D}$ requires knowledge of $S_{e d}$ which is poorly known. The recycling coefficient $R_{W}$ is even more uncertain during $N B I$ due to the complications caused by the beam ions.

The modeling in this paper gives values for $S_{\text {ed }}$ and $S_{S O}$. The value of $R_{W}$ is a parameter which can be adjusted to fit measurements. The recycling coefficient at the

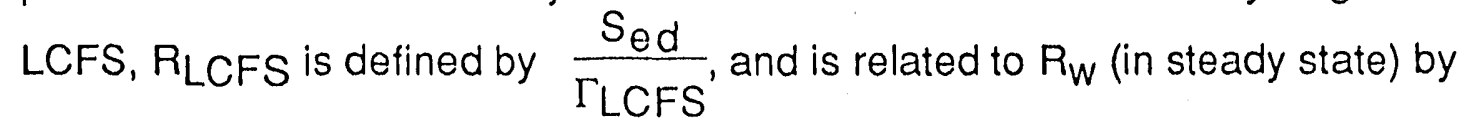

$$
R_{\text {LCFS }}=R_{W}-\frac{S_{S O}}{\Gamma_{\text {LCFS }}}\left(1-R_{W}\right)
$$

We compared results from modeling with $R_{W}=0.92$ and 0.85

\section{TRANSP Modeling}

The central plasma region for the supershot was extensively diagnosed, so the plasma parameters are well known. These measurements were used in the TRANSP modeling. Various parameters which were predicted by the model were in good agreement with other measurements. Examples are the neutron emission rate, including its radial profile, the stored energy, and the position of the peak $\mathrm{X}$-ray emission. Plasma 
profiles are shown in Figs. 3-4. TRANSP uses measured plasma profiles along with its computed particle and energy sources and sinks to compute the transport coefficients.

The TRANSP modeling extends out to the LCFS, but measurements in the plasma edge region (within about $0.1 \mathrm{~m}$ of the LCFS) are sparse. The temperature measurements were made along the horizontal midplane. The $T_{i}$ measurements from charge-exchange recombination spectroscopy extended from $R=2.5$ to $3.0 \mathrm{~m}$, and were extrapolated to the LCFS. The $T_{e}$ measurements were from electron cyclotron emission measurements. The signals extended through the plasma center and past the LCFS into the scrape off region, but the measured values in the edge and scrape off regions are unreliable since the plasma is less opaque at low density, and since the signal is low at low temperature.

The electron density profile was derived from interferometry along vertical chords. The measurements in the edge are uncertain for several reasons. The scrape off density contributes to the line integrals, and has to be subtracted from the data before Abel inversion. This contribution is poorly known. One goal of this paper is to improve our knowledge of this contribution.

\section{Edge and Scrape off modeling}

For the TRANSP modeling, the plasma parameters are extended to the LCFS. The B2 code 3 models the plasma in both the edge and scrape off regions. The bcundary between the main plasma and edge is arbitrarily set (at $r=a-0.05=0.75 \mathrm{~m}$ ). B2 computes the plasma profiles in two dimensions. The flux surfaces within the LCFS are are derived by TRANSP and those outside the LCFS are derived by an equivalentfilament analysis code. The latter code distributes axisymmetric currents to match 
measurements of the poloidal field and flux and the total plasma current. Flux contours are shown in Fig. 5.

The plasma is assumed to flow classically along the magnetic field lines and to flow perpendicular to the field lines with transport coefficients $D_{e}, \chi_{e}$, and $\chi_{i}$ which are user specified. The heat convection coefficient is assumed to be 5/2. For simplicity, we assumed $D_{e}, \chi_{e}$, and $\chi_{i}$ to be constants, extrapolating the values computed by TRANSP, as shown in Fig. 6. Also the TRANSP values of $n_{e}, T_{e}$, and $T_{i}$ at $r=0.75 \mathrm{~m}$ are used for the B2 boundary conditions. The profiles in the edge and scrape off regions at the outer midplane are shown in Figs. 7-8. These profiles very along the magnetic flux contours. We assumed that no impurities are present for the B2 and DEGAS modeling, except for computing the radiation from the edge, as is discussed below. The boundary condition for B2 at the inner limiter is that the parallel flow is at the sound speed.

The user specified fraction $R_{W}$ of the deuterium striking the limiter is emitted as neutral $D^{0}$. The ionization of these neutrals is computed approximately by $B 2$. The resulting plasma is given as an input into the DEGAS 4 code, which calculates the neutral deposition more accurately in 3 dimensions. The $D$ from the limiter is assumed to be a user specified mix of $D^{0}$ and $D_{2}$. This is taken to be $100 \% D_{2}$ in this study. The DEGAS source distribution is input back into $\mathrm{B} 2$, and the process is process is iterated to improve the accuracy of the model. 5 One goal is to provide an accurate estimation of the $S_{\text {ed }}$. which is input into TRANSP. 6 Results are given in Table 1.

There are several tests of the accuracy of the B2 / DEGAS modeling. The B2 plasma profiles imply energy and particle fluxes. These are compared with the values computed by TRANSP in Table $I I$ and in Figs. $9-10$. The TRANSP profile for the particle flux shows a rapid increase between $x=0.9$ and the LCFS at $x=1$. This may indicate that 
the boundary between the main plasma and edge regions should be taken closer to $x=$ 0.9. B2 also computes the particle and energy fluxes to the inner limiter. The predicted [pwer profiles are shown in Fig. 11. The profile of the temperature increase in the limiter after the discharge is measured by an array of approximately 100 thermocouples. ${ }^{7}$ The toroidal average of the data is shown in Fig. 12. From this, we infer the power flow to the limiter, assuming it is dominated by steady state flow during the NBI. The estimated total power for this supershot is $5 \mathrm{MW}$. The value predicted by the B2 / DEGAS model is four times too high. This discrepancy is discussed in the next section.

Another check of the ion flux to the limiter is given by the $D_{\alpha}$ emission. This is measured along five sightlines in TFTR. 8 DEGAS also simulates the $D_{\alpha}$ emission along the sightlines, which can be compared. The result is shown in Fig. 13. The experimental uncertainty of the measurements is $15 \%$, so the prediction is in qualitative, but not quantiative agreement.

No probe measurements were made in the scrape off region of this or similar supershots. Measurements were made in TFTR discharges with the limiter less well conditioned and with lower NBI power (up to $20 \mathrm{MW}$ ). ${ }^{9}$ The measured values of $n_{e}$ and $\mathrm{T}_{e}$ near the outer midplane, $0.125 \mathrm{~m}$ from the LCFS are indicated in Figs. 7 - 8.

\section{Discussion}

Our results give values of the edge ionization rate $S_{e d}$ comparable to those derived from simpler fits based on the SNAP analysis of TFTR discharges. 5 There, a conversion factor from the measured $D_{\alpha}$ values to $S_{e d}$ was discussed. The B2 / DEGAS modeling gives a similar value. 
We used two values for the wall recycling coefficient $R_{W}$ and compared the results with measurements to see if one gave a better fit. The predicted power loading (Fig. 11) and the $D_{\alpha}$ profile (Fig. 13) were comparable for $R_{W}=0.7$ and 0.9 , so we could not distinguish these.

The B2 / DEGAS modeling predicts a power loading on the limiter that is four times higher than that implied by thermocouple measurements. A discrepancy in the power balance is typically noticed in NBI heated discharges in TFTR. We investigated two improvements in the modeling to see if they would lower the predicted power flow. One was an estimate of power loss by radiation. Large amounts of radiation are inferred in the edge of this and other supershots from bolometry measurements. The dominant impurity identified in the main plasma is carbon which is not suprising since the limiter is made of 2600 pounds of carbon. We estimated the carbon light emission adding into B2 an empirical fit to measurements. 10 The estimate is proportional to $\mathrm{ne}^{2} / \mathrm{T}_{e}{ }^{1.5}$. We do not know the carbon concentration or $Z_{\text {eff }}$ of the edge or scrape off regions, but if $50 \%$ of the ions were carbon and $50 \%$ deuterium, then the radiation would reduce the electron power by $5 \mathrm{MW}$. This is not enough loss for agreement.

The other iriprovement we studied was modeling the effects of charge exchange of recycled neutrals with the beam ions. This increases the particle flux to the limiter. The beam ion density and average energy is calculated by TRANSP. These results were used in the DEGAS calculation, and the effects are predicted to be small for this discharge. 


\section{Acknowledgements}

We wish to acknowledge the US DOE contract \# DE-AC02-76-CHO-3073 for supporting this research.

\section{References}

1)J.A. Snipes, et al., "Wall Conditioning with Impurity Pellet Injection on TFTR", submitted at this conference.

2) R.V. Budny, et al., Nucl. Fusion 32 (1992) 419.

3) B. J. Braams, "Computational Studies in Tokamak Equilibrium and Transport", Ph. D. Thesis (1986).

4) D.H. Heifetz, et al, J. Comput. Phys. 46 (1982; 309.

5) D, Stotler, et al., submitted at this conference.

6) R.V. Budny and the TFTR group, J. Nucl. Mat. 176-177 (1990) 427-431.

7) A.C. Janos, M. Corneliussen, E. Fredrickson, K.M. McGuire, K. Owens, and M. Ulrickson, Rev. Sci. Instrum. 61 (1990) 2973-2975.

8) A. Ramsey and S.L. Turner, Rev. Sci. Instrum. 58 (1987) 1211.

9) D.M. Manos, S.J. Kilpatrick, M.G. Bell, R.V. Budny, and M. Ulrick'son, J. Nucl. Mat. 162164 (1989) 251-257.

$10)$ Ralf Schneider, private communication. 


\section{Table I}

\section{Ionization rates from the modeling $\left(R_{W}=0.9\right)$}

$\begin{array}{cccc} & \text { maln } & \text { edge } & \text { scrape off } \\ r(m) & 0-0.75 & 0.75-0.80 & >0.80\end{array}$

$\begin{array}{lllll}\text { Recycling } D^{+} \text {source rate } & (1022 / \mathrm{sec}) & 0.28 & 2.28 & 4.24 \\ \text { Beam } D^{+} \text {source rate } & \left(10^{22} / \mathrm{sec}\right) & 0.15 & 0.0 & 0.0\end{array}$


Table II

Energy and particle fluxes from the modeling $\left(R_{W}=0.9\right)$

TRANSP

B2 / DEGAS

$r(m)$

0.75

0.75

80

limiter

conducted ion power (MW) $\quad 13.0$

convected ion power (MW) $\quad 1.7$

total ion power

$(\mathrm{MW})$

14.7

17.50

18.68

11.84

conducted el power (MW) 3.2

convected el power (MW) 1.0

total el power

$(\mathrm{MW}) \quad 4.2$

8.66

4.14

8.58

Do flux in

$\left(10^{22} / \mathrm{sec}\right) \quad 0.0$

0.28

3.10

7.34

$D+$ flux out

$\left(10^{22} / \mathrm{sec}\right) \quad 0.4$

0.84

3.77

7.94

$R \equiv \frac{D^{0} \text { flux in }}{D+\text { flux out }}$

0.822

0.925 


\section{Figures Captions}

1) Number of particles within the LCFS and the edge recycling rate in a supershot. The electron density profile $n_{\theta}(x, t)$ is symmetrizing from the measured (Abel inverted) profile. The impurity density, $n_{i m p}$, is calculated from $Z_{e f f}$, which can be calculated from a visible bremsstrahlung measurement. The beam density, $n_{\text {beam }}$, is calculated from the Monte Carlo neutral beam deposition. The edge recycling rate is given by the B2 / DEGAS modeling.

2) Schematic of the plasma regions, $D^{0}$ source rates within them, and $D^{+}$fluxes between them.

3) Plasma density profiles at $3.7 \mathrm{sec}$ from the TRANSP modeling.

4) Plasma temperature profiles at $3.7 \mathrm{sec}$ from the TRANSP modeling.

5) Magnetic flux surfaces. The contours within the LCFS are from the TRANSP modeling with equal spacing of the square root of the normalized tcroidal flux. The contours in the scrape off region are unit increments of poloidal flux calculated with an equivalentfilament analysis code.

6) Profiles of the power conducted and convected via electrons and lons. Power losses via radiation and charge-exchange are small, and not shown.

7) Detailed view of the plasma density profiles along the outer midplane from the TRANSP and B2 modeling. The probe result is from a different discharge with relatively high recycling and $20 \mathrm{MW}$ of $\mathrm{NBI}$ power.

8) Detailed plasina temperature profiles along the outer midplane from the TRANSP and B2 modeling. The probe result is from a different discharge with relatively hight recycling and $20 \mathrm{MW}$ of $\mathrm{NBI}$ power.

9) Profiles of the transport coefficients measured by TRANSP within the LCFS and those assumed for the B2 modeling in the edge and scrape off regions.

10) Profile of the electron particle flux from the TRANSP modeling. 
11) Predicted electron and ion power flow to the limiter.

12) Profile of the average temperature increase on the inner limiter during the discharge versus the poloidal angle.

13) Profile of the measured $D_{\alpha}$ along five sightlines through the discharge and the predicted values from the B2 / DEGAS modeling. 
Particles within the last closed flux surface and the edge recycling rate

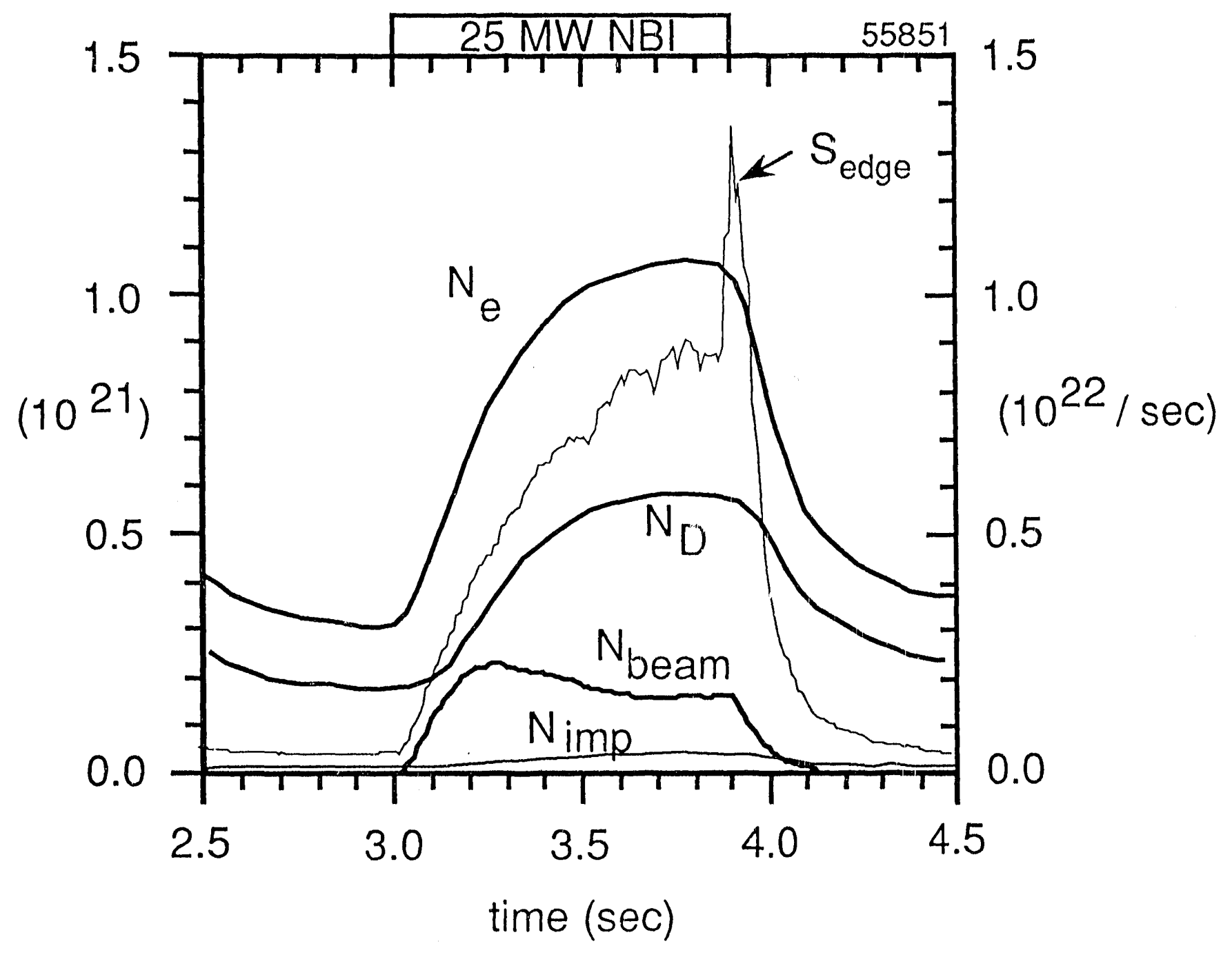

Figure 1 
Plasma regions
$D^{0}$ source
Main
$D^{+}$flux
$\Gamma_{m}^{+}$
$\Gamma_{\text {LCFS }}^{+}$
$\Gamma_{w}^{+}$

Figure 2 
Density profiles from TRANSP

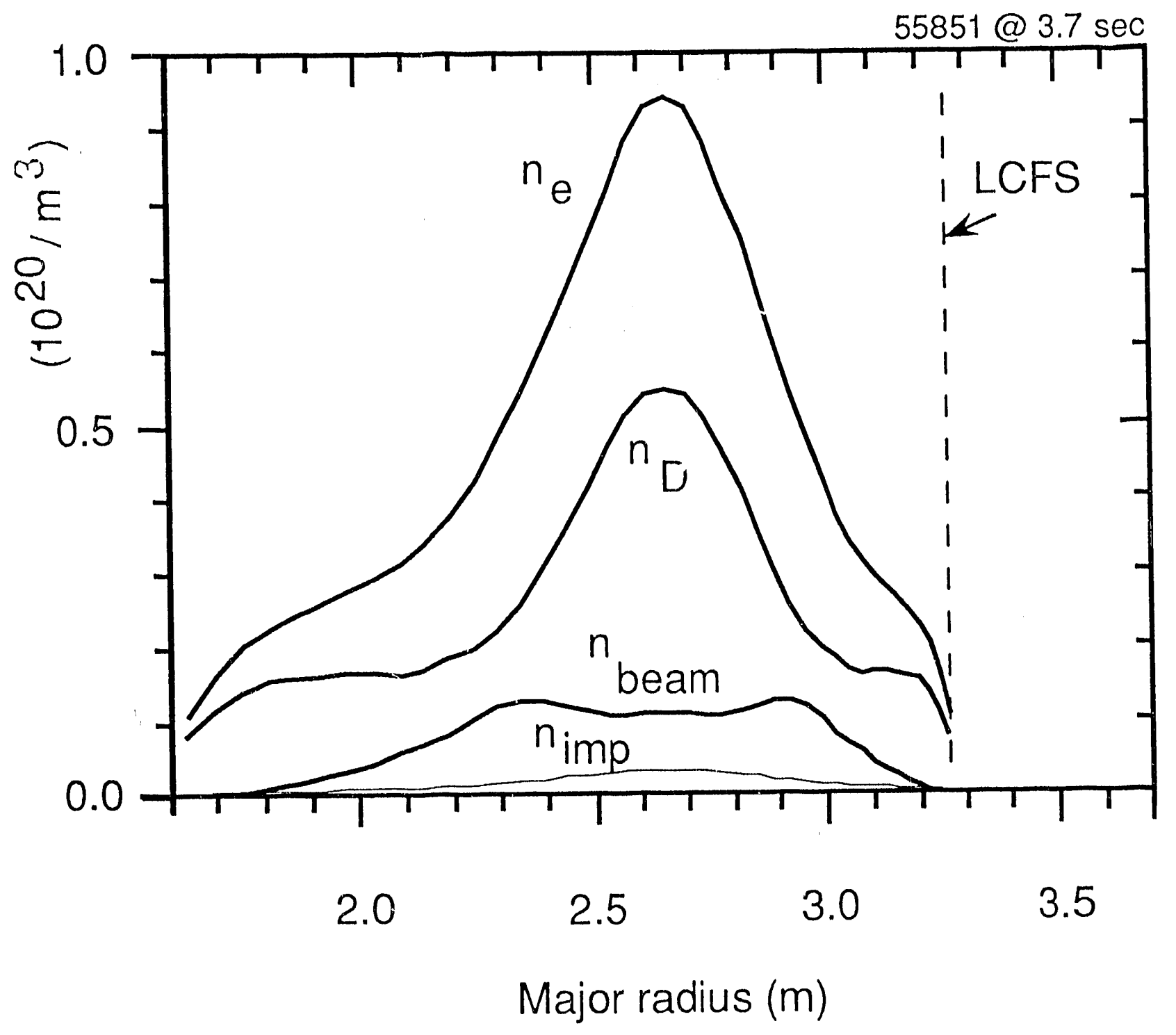

Figure 3 


\section{Temperature profiles from TRANSP}

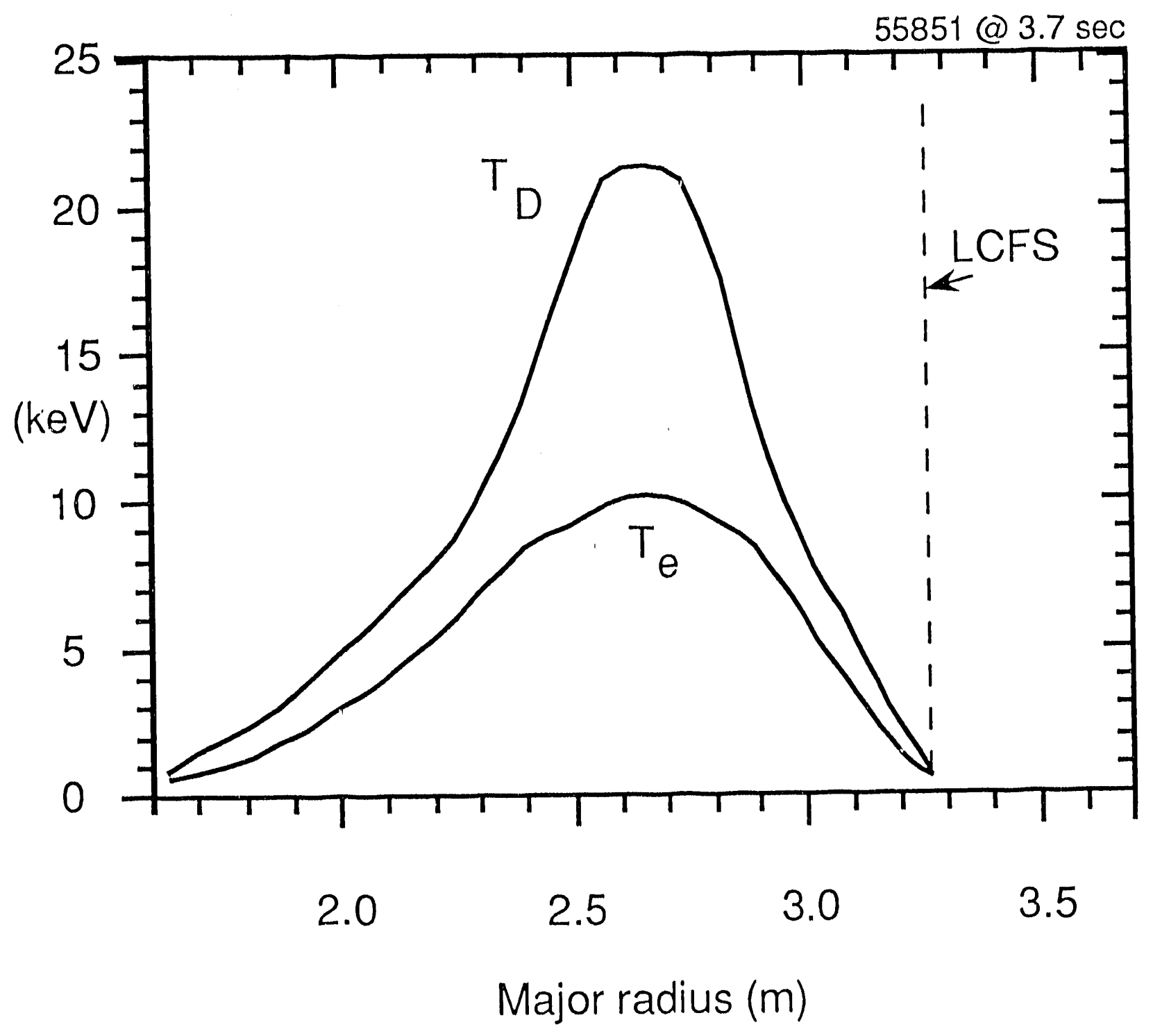

Figure 4 


\section{Magnetic flux surfaces}

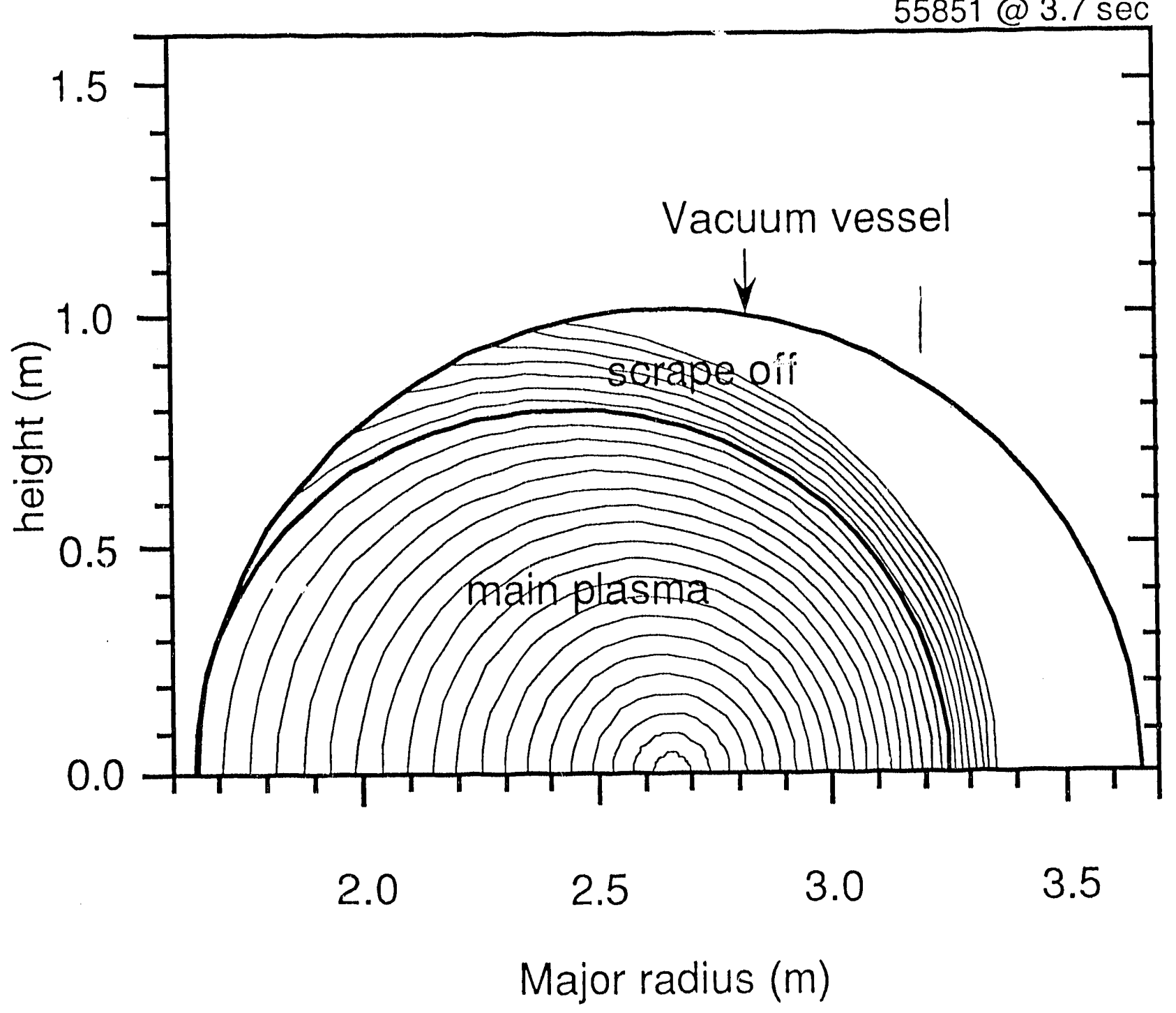

Figure 5 


\section{Transport coefficients}

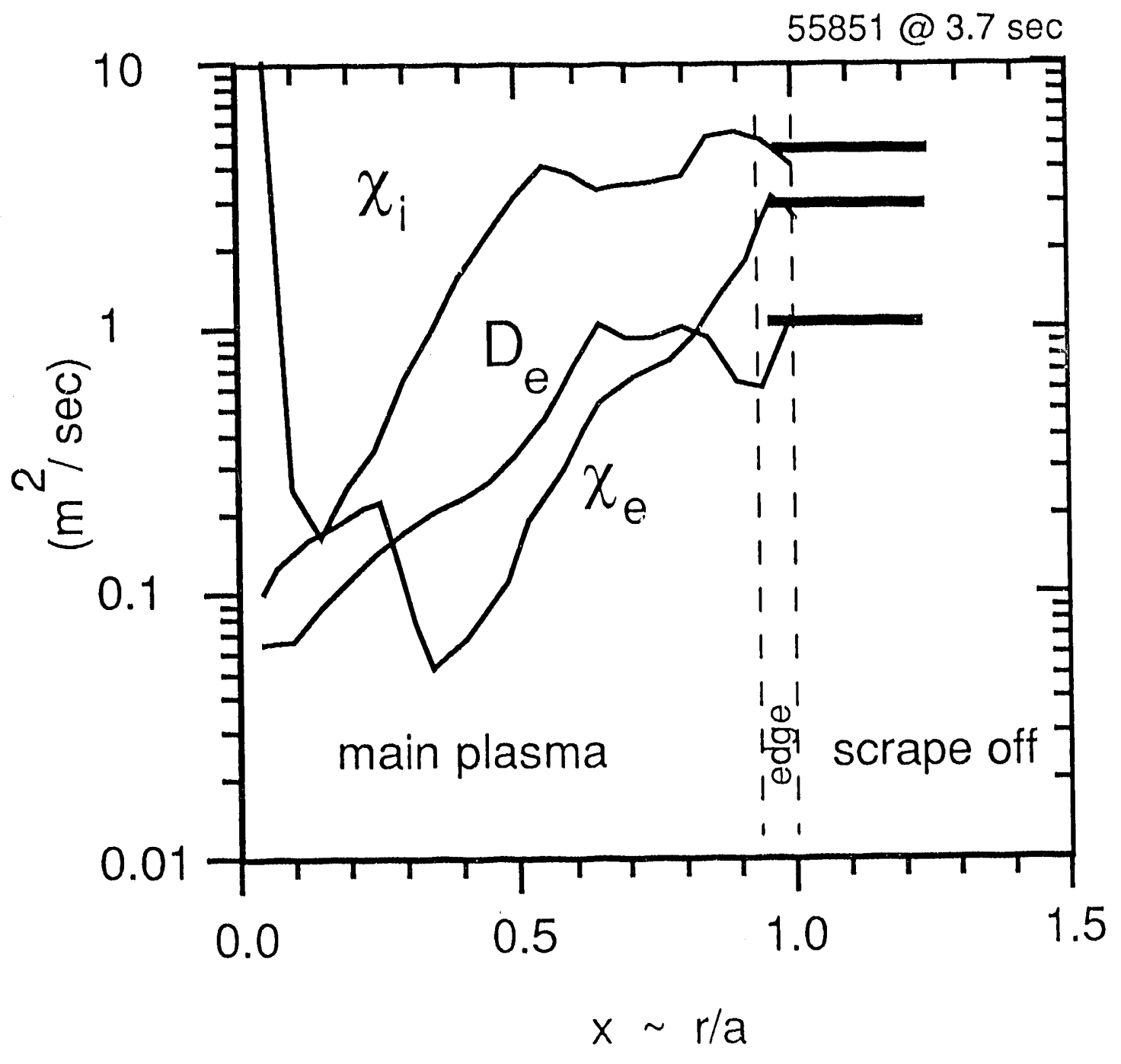

Figure 6 
Edge density profiles

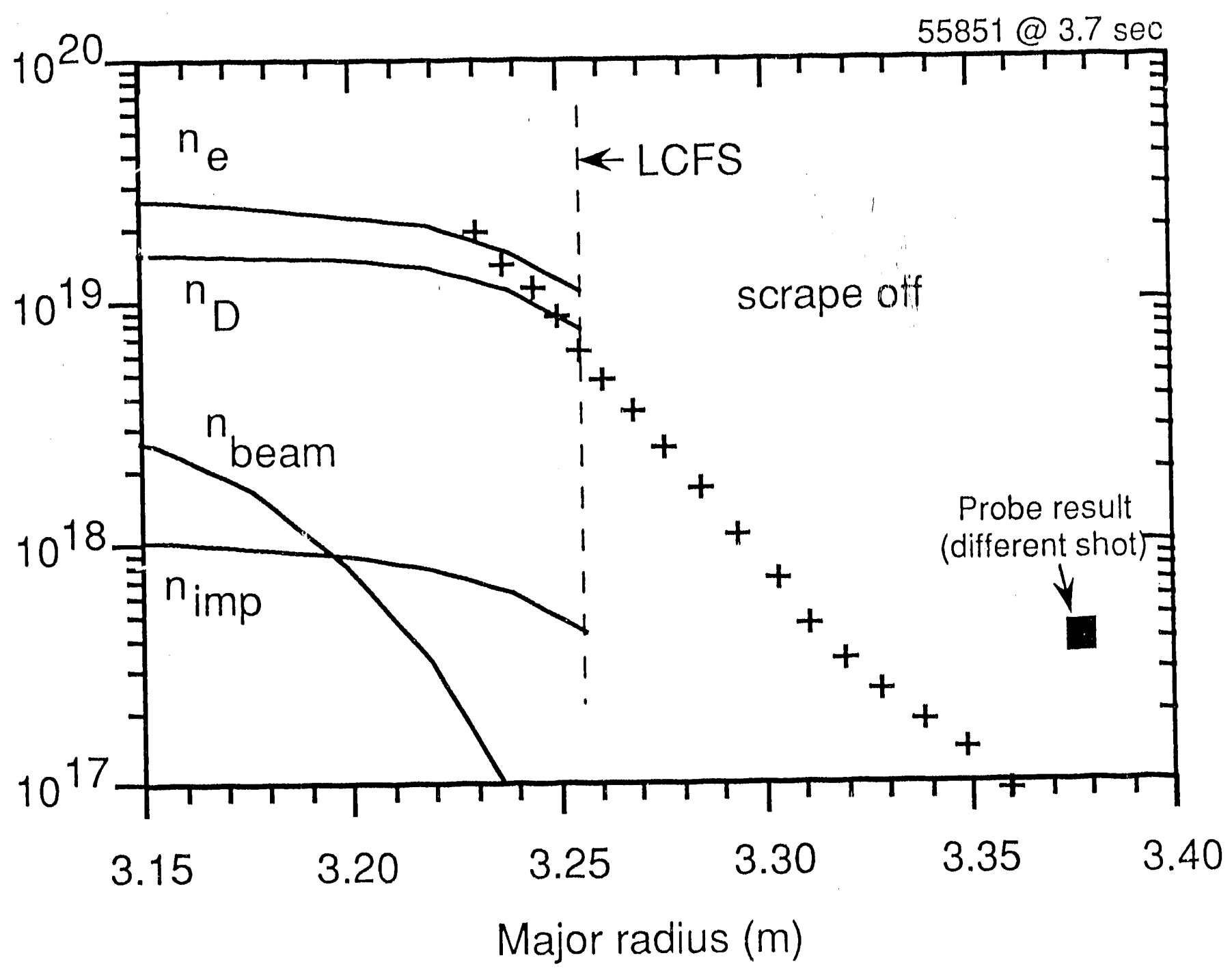

Figure 7 
Edge temperature profiles

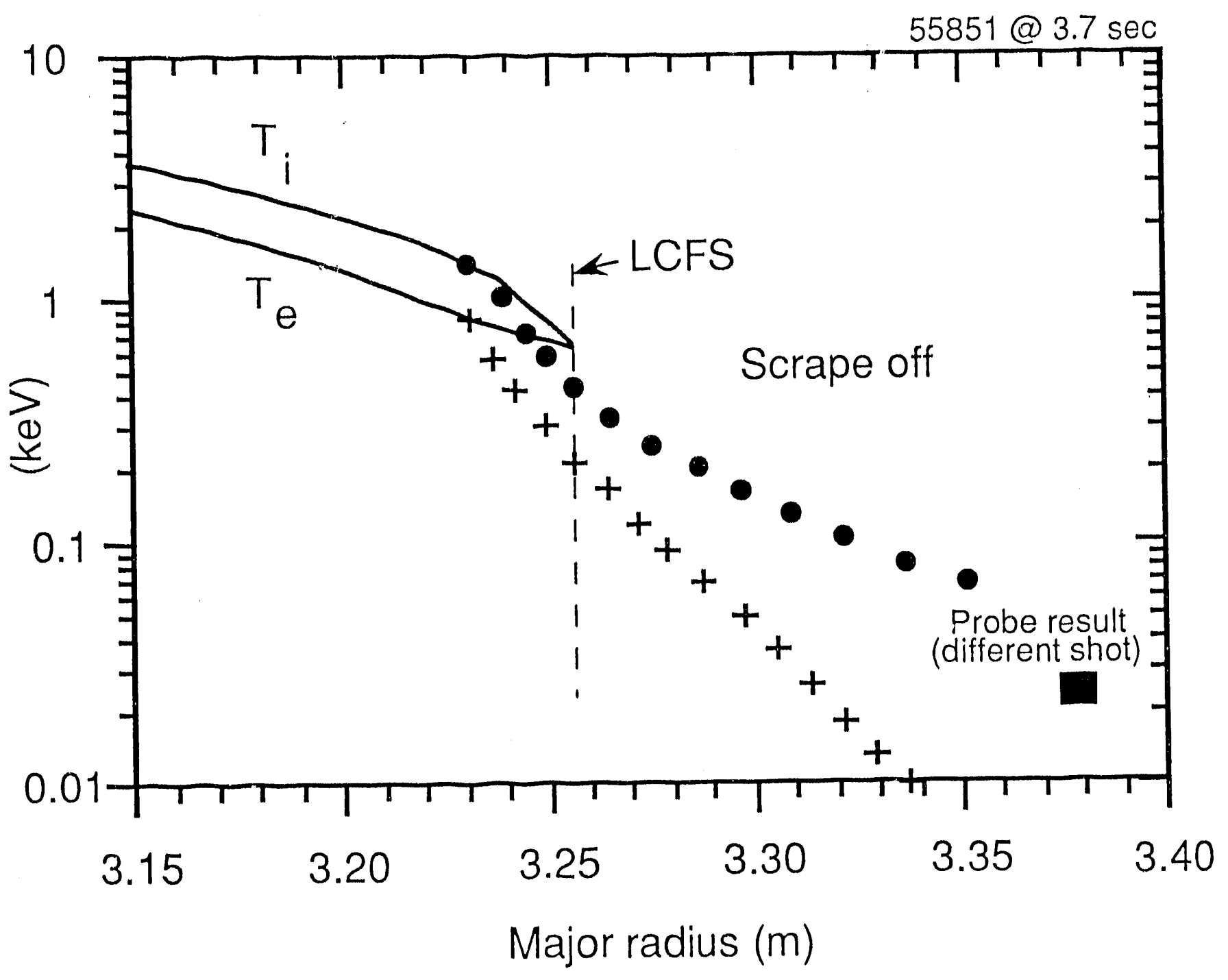

Figure 8 


\section{Power fluxes}

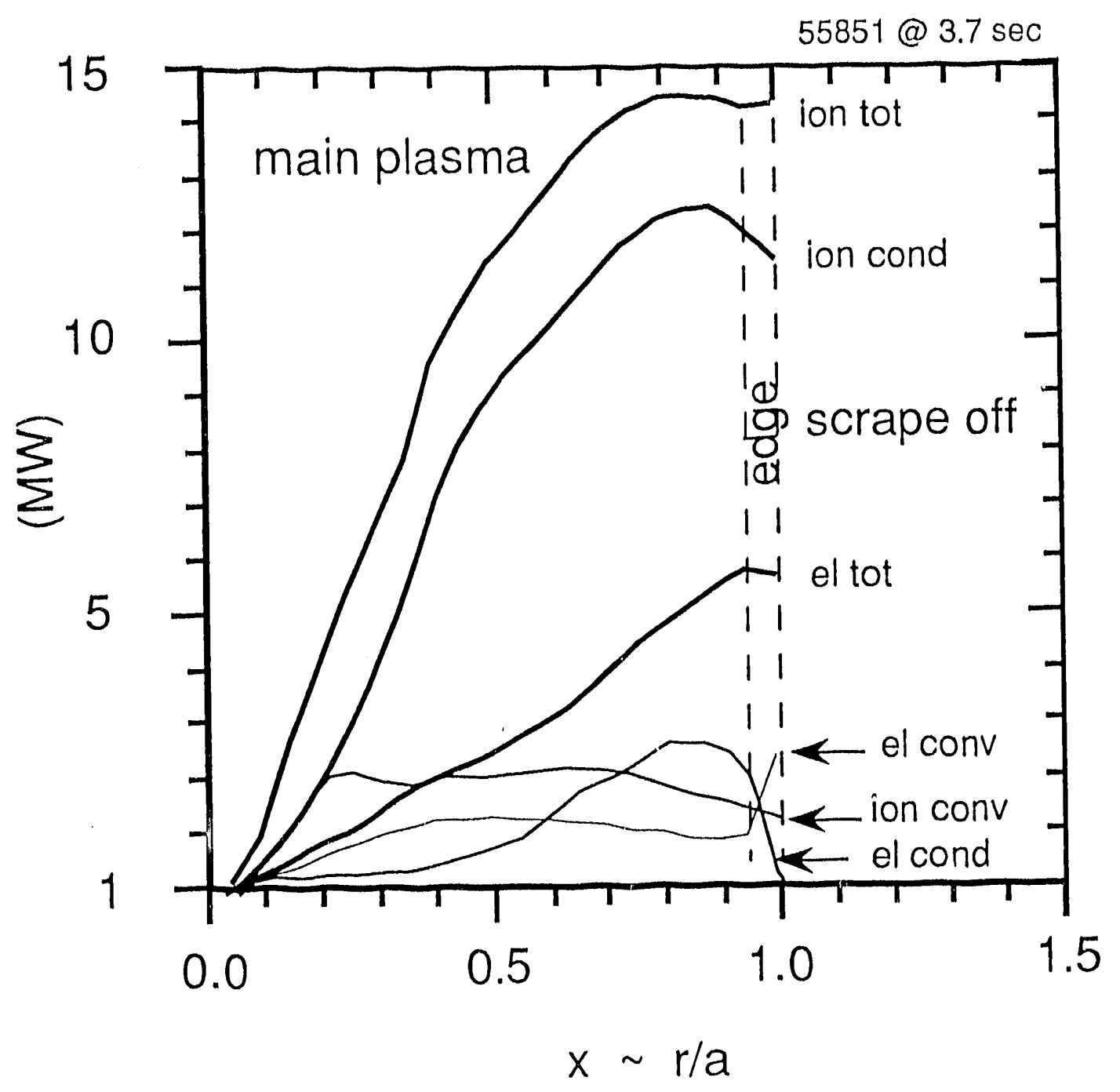

Figure 9 
Electron particle flux

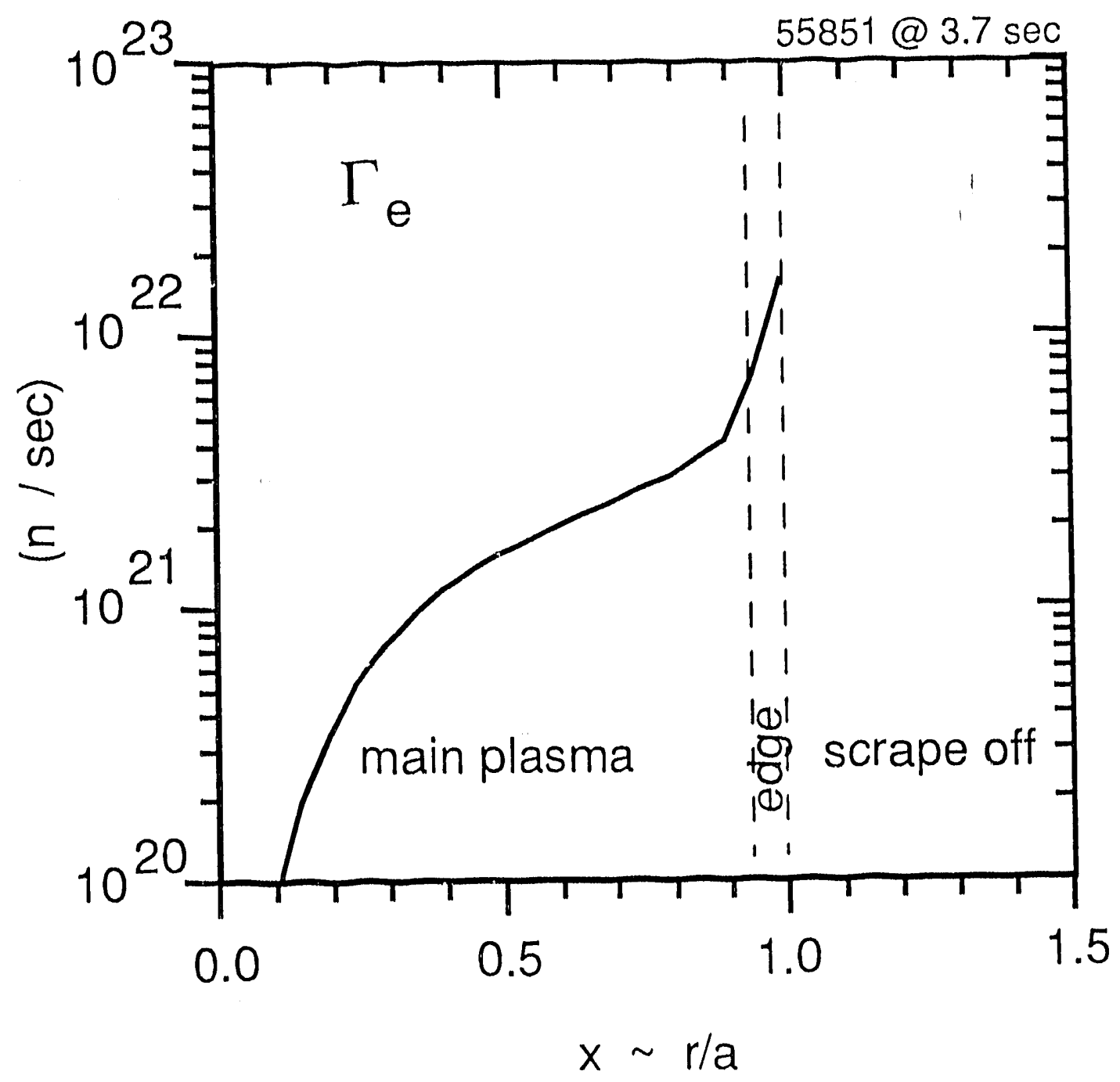

Figure 10 


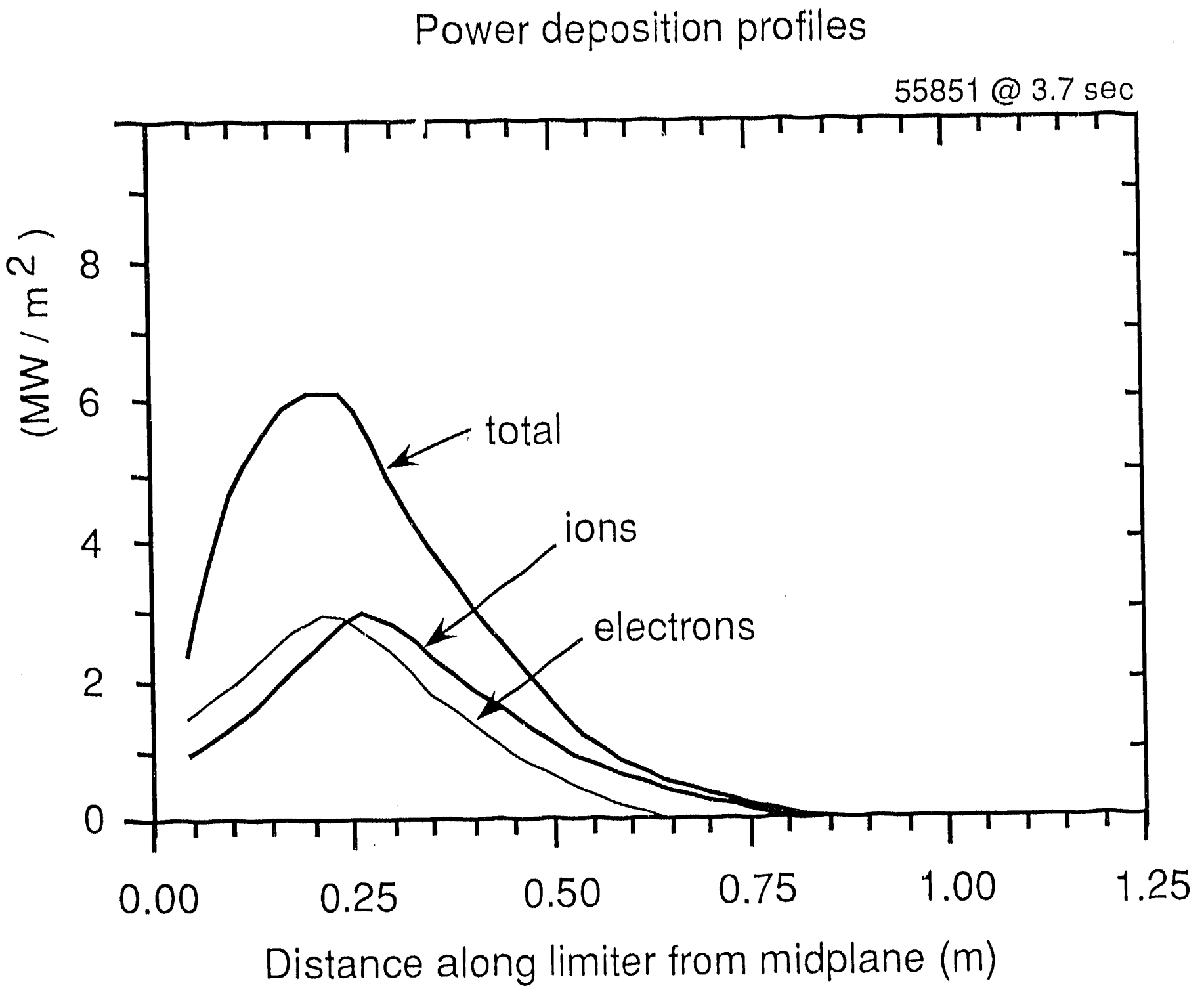

Figure 11 


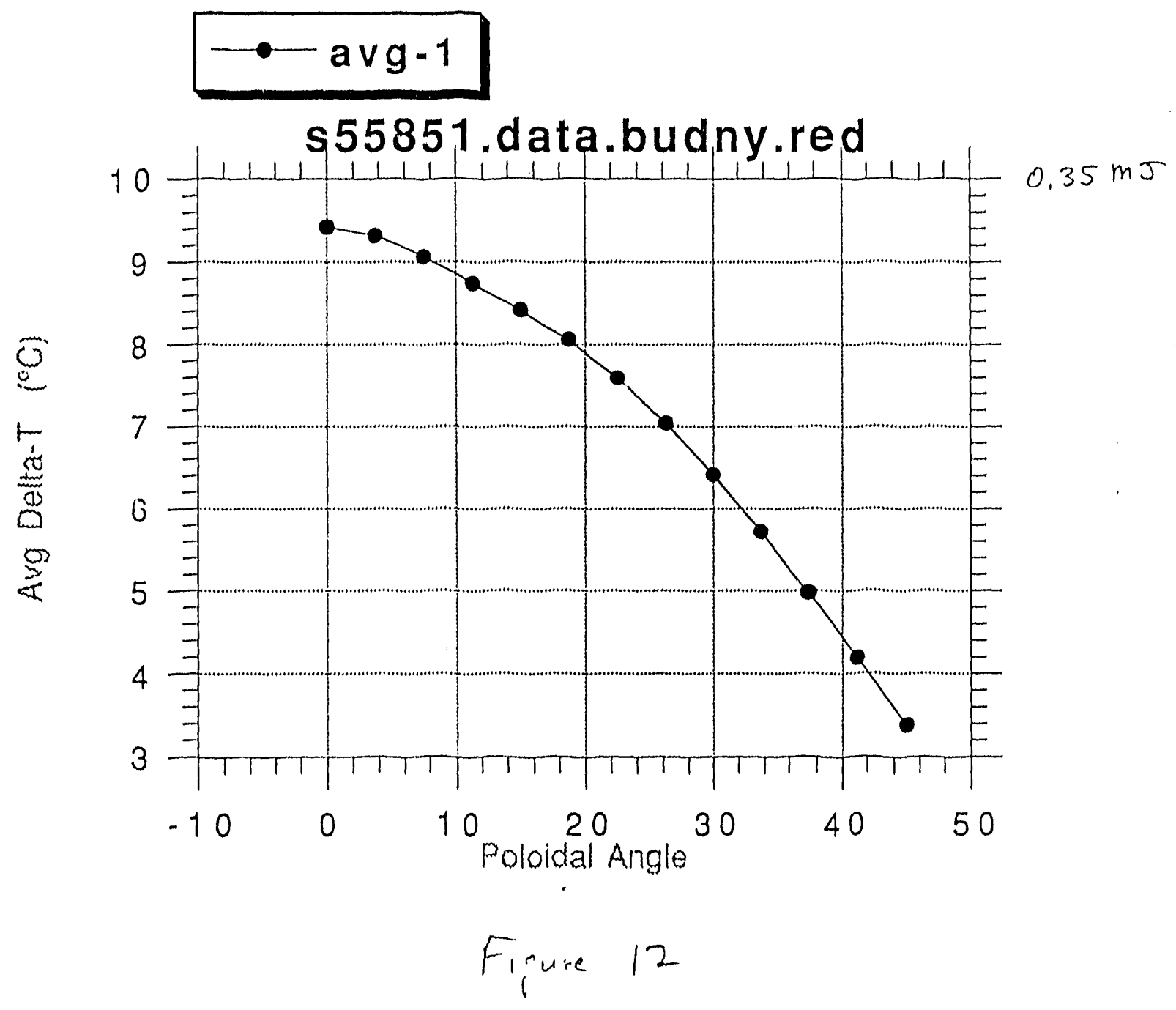




\section{Measured $D_{\alpha}$ and prediction from B2 / DEGAS modeling}

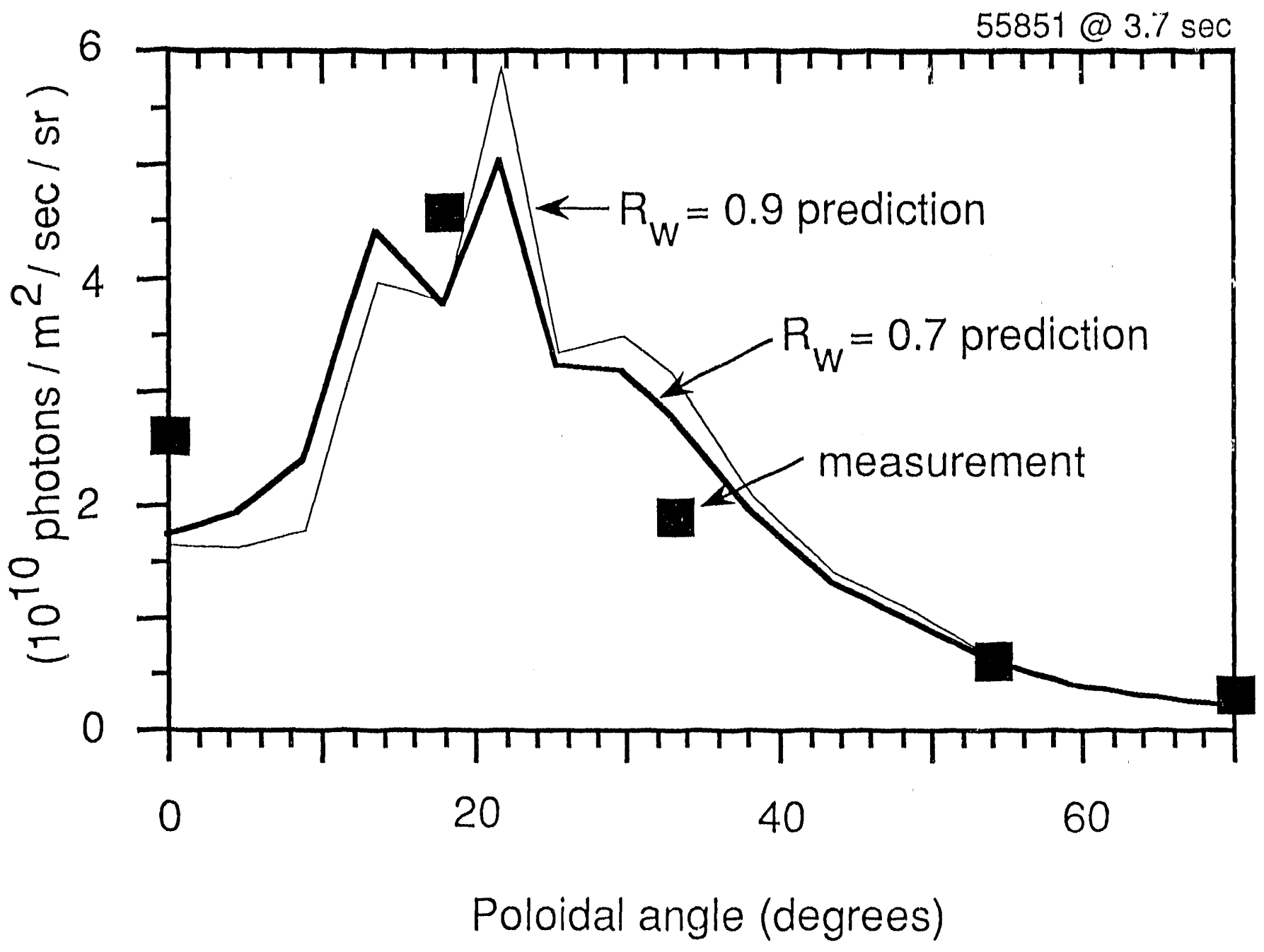

Figure 13

\section{DISCLAIMER}

This report was prepared as an account of work sponsored by an agency of the United States Government. Neither the United States Government nor any agency thereof, nor any of their employees, makes any warranty, express or implied, or assumes any legal liability or responsibility for the accuracy, completeness, or usefulness of any information, apparatus, product, or process disclosed, or represents that its use would not infringe privately owned rights. Reference herein to any specific commercial product, process, or service by trade name, trademark, manufacturer, or otherwise does not necessarily constitute or imply its endorsement, recommendation, or favoring by the United States Government or any agency thereof. The views and opinions of authors expressed herein do not necessarily state or reflect those of the United States Government or any agency thereof. 

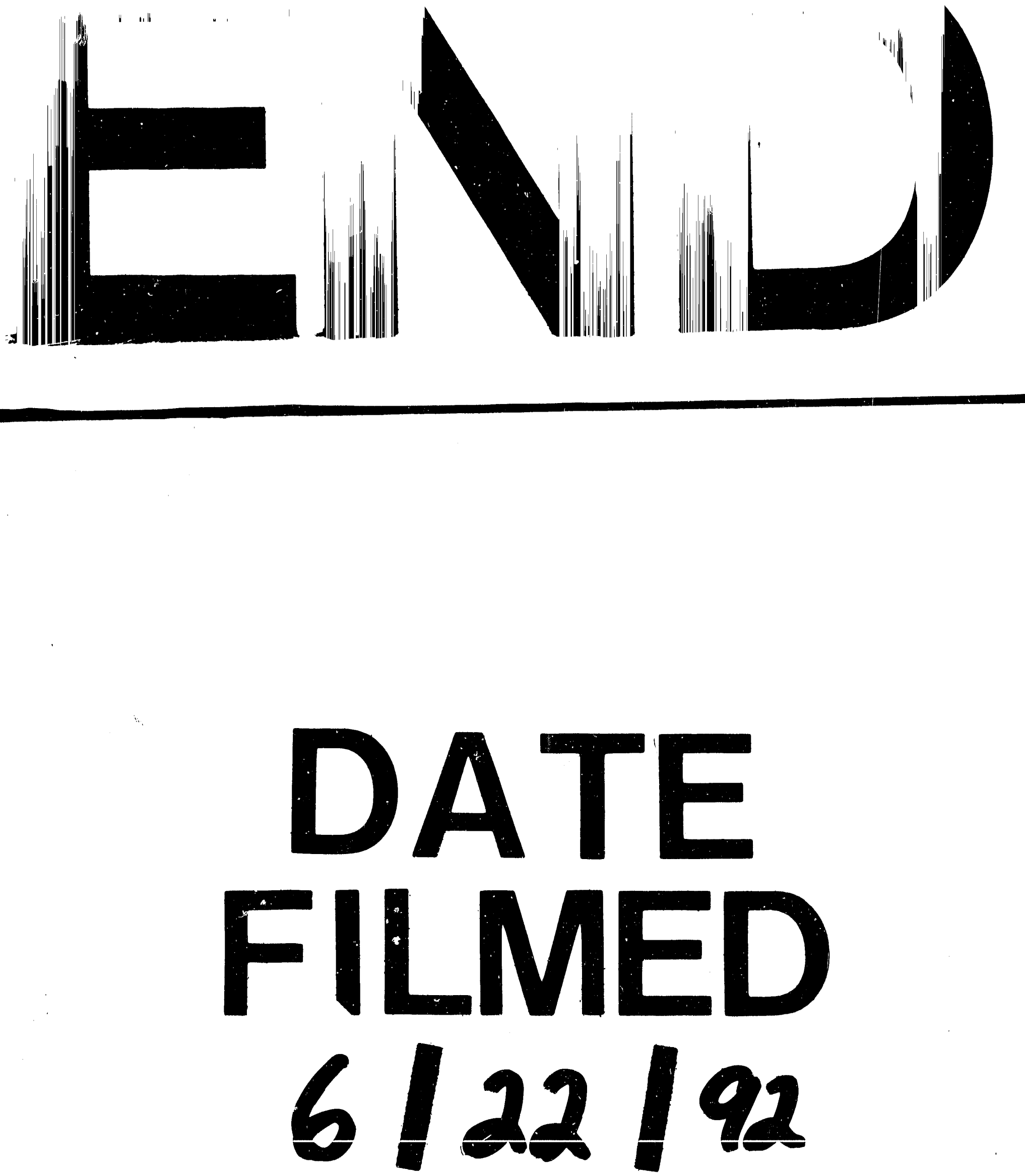
